\title{
Tris-(8-hydroxyquinoline) aluminum nanoparticles prepared by vapor condensation
}

\author{
J.-J. Chiu and W.-S. Wang ${ }^{\text {a) }}$ \\ Department of Electrical Engineering, National Taiwan University, Taipei, Taiwan 106, Republic of China \\ C.-C. Kei and T.-P. Perng ${ }^{\text {b) }}$ \\ Department of Materials Science and Engineering, National Tsing Hua University, Hsinchu, Taiwan 300, \\ Republic of China
}

(Received 3 September 2002; accepted 13 May 2003)

\begin{abstract}
Tris-(8-hydroxyquinoline) aluminum $\left(\mathrm{AlQ}_{3}\right)$ spherical nanoparticles of the average size varying from 50 to $500 \mathrm{~nm}$ were synthesized by vapor condensation. The surface of the nanoparticles is quite sleek and smooth like that of pearls. The X-ray diffraction patterns reveal that the nanoparticles have an amorphous structure. The chemical bonding of $\mathrm{AlQ}_{3}$ is preserved in the nanoparticles even after evaporation at $410{ }^{\circ} \mathrm{C}$. The photoluminescence spectra of the nanoparticles show a broadened peak varying from 4500 to $7000 \AA$, with the maximum intensity at about $5380 \AA$. The maximum intensity increases as the particle size decreases, owing to the large specific surface area. (C) 2003 American Institute of Physics. [DOI: 10.1063/1.1591249]
\end{abstract}

In the past decades, the trend of technological development in electronics has been to make the device smaller and more powerful. This requires that each of the constituent materials be operated more efficiently and cost effectively. Since the discovery of the buckyball ${ }^{1}$ and carbon nanotubes, ${ }^{2}$ a great number of nanostructures such as nanoparticles, nanowires, and nanorods have been produced. The prescribed size, composition, and homogeneity should be well controlled to explore new applications such as field emission sources, ${ }^{3}$ single electron transistors, ${ }^{4}$ chemical and biological sensors, ${ }^{5}$ quantum dot lasers, ${ }^{6}$ and other optoelectronic devices. $^{7}$ Though the nanoparticles for light emitting devices have been investigated for many years, they are all made of inorganic materials. Inorganic nanoparticles can be prepared by various methods such as chemical vapor deposition ${ }^{8}$ or molecular beam epitaxy, ${ }^{9}$ by which the nanoparticles are fabricated at high temperatures and controlled by the lattice mismatch. ${ }^{10}$ Since the first efficient electroluminescence of organic light emitting diode (OLED) with a low driving voltage was reported by Tang and VanSlyke in $1987,{ }^{11}$ tris- $(8-$ hydroxyquinoline) aluminum $\left(\mathrm{AlQ}_{3}\right)$ has become one of the most important emitting materials for the OLED devices. It is then of great interest to prepare organic nanostructures due to many properties that may offer applications in nanooptoelectronic devices. ${ }^{12,13}$ In this work, the $\mathrm{AlQ}_{3}$ nanoparticles were first synthesized by vapor condensation. The surfaces of the nanoparticles are found quite sleek and smooth like those of pearls. The photoluminescence (PL) spectra of $\mathrm{AlQ}_{3}$ nanoparticles with different sizes are also measured for comparison.

The experimental setup for the vapor phase condensation of $\mathrm{AlQ}_{3}$ is shown in Fig. 1. The $\mathrm{AlQ}_{3}$ powder (TCI Ltd., T1527) was placed in a graphite boat, and the silicon substrate was placed under a liquid nitrogen-cooled trap. The

\footnotetext{
a) Author to whom correspondence should be addressed; electronic mail: wswang@cc.ee.ntu.edu.tw

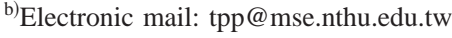

distance between the graphite boat and the substrate $d$ was adjustable. An argon flow of pressure $P_{\text {Ar }}$ was then launched into the chamber. The temperature of the boat $T$ was regulated by a power supply and a K-type thermocouple. $\mathrm{AlQ}_{3}$ nanoparticles were grown directly on the silicon substrate. The particle size was found to depend on $P_{\mathrm{Ar}}, T$, and $d$.

Figure 2 shows the field emission gun scanning electron microscopy (FEGSEM) images of $\mathrm{AlQ}_{3}$ nanoparticles. For instance, the size of $\mathrm{AlQ}_{3}$ nanoparticles fabricated at $P_{\mathrm{Ar}}$ $=133 \mathrm{~Pa}, T=410^{\circ} \mathrm{C}$, and $d=10 \mathrm{~cm}$ is about $50-100 \mathrm{~nm}$, as shown in Fig. 2(a). As can be seen from the figure, the spherical particles tend to cluster together as reticular networks. At a higher pressure $P_{\mathrm{Ar}}=4 \mathrm{kPa}$ but with the same $T$ and $d$, the size of $\mathrm{AlQ}_{3}$ nanoparticle is increased to about 100-300 nm, as displayed in Fig. 2(b). When the pressure is increased even higher, e.g., $P_{\mathrm{Ar}}=13.3 \mathrm{kPa}$ and the distance is increased to $d=20 \mathrm{~cm}$, most nanoparticles become isolated and have an average size of about $500 \mathrm{~nm}$. The surface is quite sleek and smooth like that of pearls, as shown in Fig 2(c). Moreover, the $\mathrm{AlQ}_{3}$ nanoparticles remain stable in the FEGSEM for more than $3 \mathrm{~h}$ without any change of the surface morphology, indicating an excellent thermal stability.

The experimental results shown in Figs. 2(a)-2(c) indicate that the size and configuration of the particles are dependent on the argon pressure $P_{\mathrm{Ar}}$, the distance $d$, and the

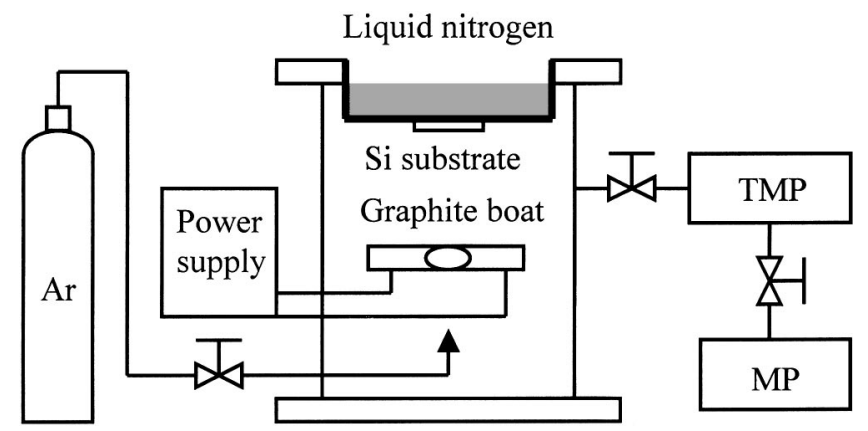

FIG. 1. Experimental setup of the vapor condensation system. 


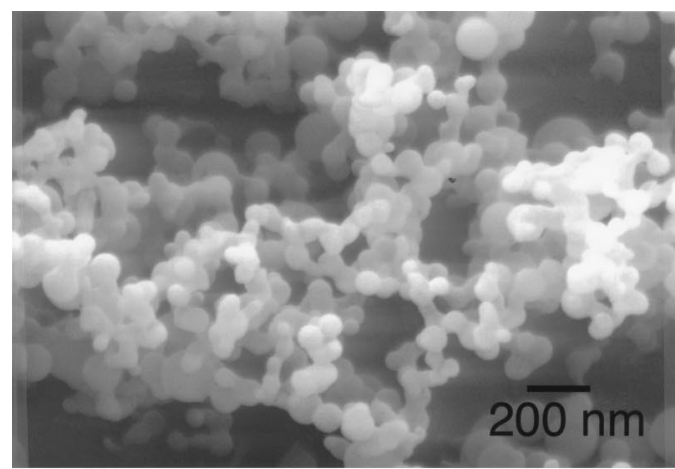

(a)

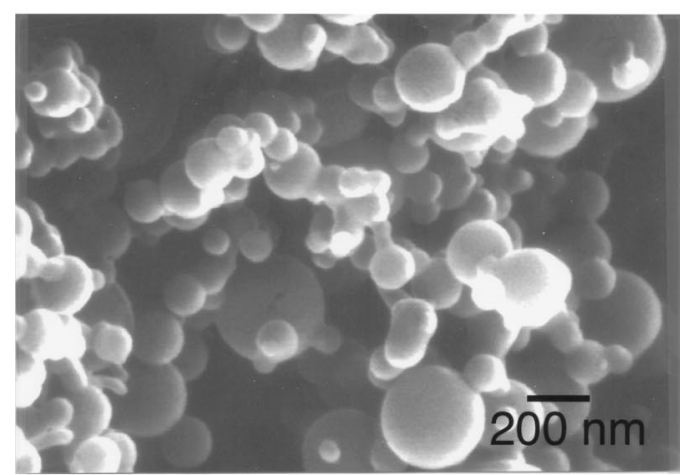

(b)

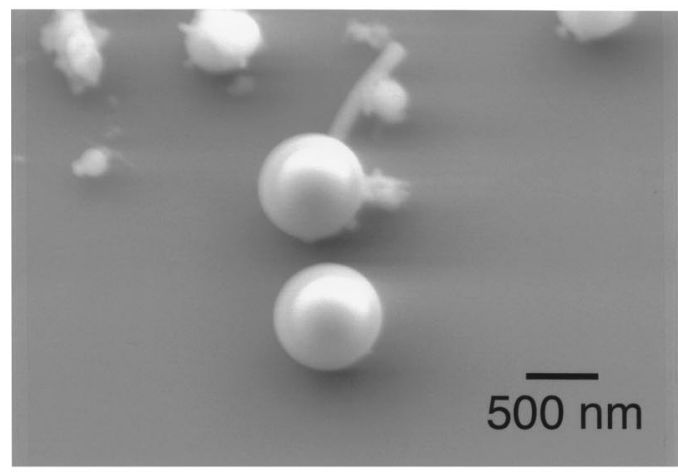

(c)

FIG. 2. FEGSEM images of $\mathrm{AlQ}_{3}$ nanoparticles prepared under different conditions. (a) $P_{\mathrm{Ar}}=133 \mathrm{~Pa}, T=410^{\circ} \mathrm{C}$, and $d=10 \mathrm{~cm}$, (b) $P_{\mathrm{Ar}}=4 \mathrm{kPa}$, $T=410^{\circ} \mathrm{C}$, and $d=10 \mathrm{~cm}$, and (c) $P_{\mathrm{Ar}}=13.3 \mathrm{kPa}, T=400{ }^{\circ} \mathrm{C}$, and $d$ $=20 \mathrm{~cm}$.

boat temperature $T .^{14,15}$ For simplicity, the boat temperature $T$ is set at $410{ }^{\circ} \mathrm{C}$, and the dependences of particle size on $P_{\mathrm{Ar}}$ and $d$ are considered separately. At fixed $T$ and $d$, the average size of particles increases significantly and the range of size distribution becomes larger when $P_{\mathrm{Ar}}$ increases, as shown in Fig. 3(a). Similarly, at fixed $T$ and $P_{\text {Ar }}$, the average size also increases when $d$ increases, as shown in Fig. 3(b). In general, the boat temperature should be controlled at $400-410{ }^{\circ} \mathrm{C}$ in order to form the nanoparticles when $P_{\mathrm{Ar}}$ is higher than $133 \mathrm{~Pa}$. When the pressure is lower than $133 \mathrm{~Pa}$, the boat temperature must be carefully controlled. For example, when $P_{\mathrm{Ar}}=13.3-133 \mathrm{~Pa}$, nanoparticles can be obtained only at $T=405-410^{\circ} \mathrm{C}$.

Although the dependence of the particle size on $P_{\mathrm{Ar}}$ and $d$ has been reported for inorganic nanoparticles, ${ }^{14}$ it is surprising that the $\mathrm{AlQ}_{3}$ molecule with a molecular weight much larger than that of Ar atom also exhibits similar behav-
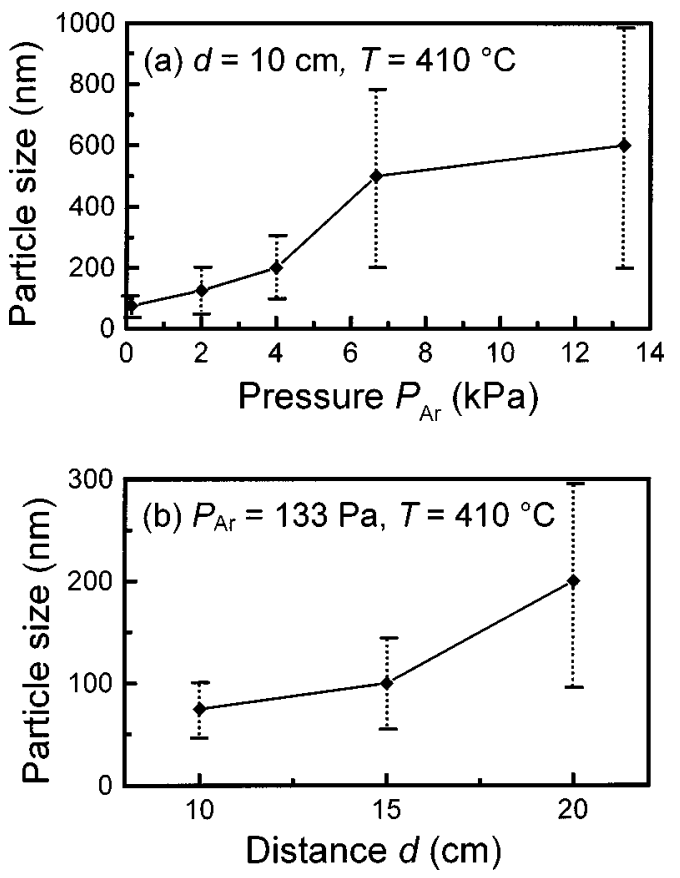

FIG. 3. (a) Relationship between the particle size and argon pressure $P_{\mathrm{Ar}}$, and (b) relationship between the particle size and separation distance $d$.

ior. This can be understood by the collision mechanism during the evaporation. The $\mathrm{AlQ}_{3}$ molecules collide with the $\mathrm{Ar}$ atoms and gradually lose their energy. Since the substrate is cooled down to $-196^{\circ} \mathrm{C}$ by liquid nitrogen, it is believed that this temperature is low enough to stop nanoparticles from coalescing on the substrate. The particle size is related to the energy loss, which can be realized by the ratio between the atomic weights of evaporated material and $\mathrm{Ar}^{15}$ For those inorganic materials whose atomic weights are comparable to that of Ar, their kinetic energy can be reduced by colliding with the Ar atoms. For instance, the atomic masses of $\mathrm{Si}$ and $\mathrm{Ar}$ are 28 and 40, respectively. The ratio of the atomic masses is 0.7 . The molecular weight of $\mathrm{AlQ}_{3}$ is 459 , and the ratio becomes about 11.5. This value is much greater than that for $\mathrm{Si}$, and the particle size should be larger under the same condition. ${ }^{15}$ Previously, the sizes of Si nanoparticles prepared at $P_{\mathrm{Ar}}=133 \mathrm{~Pa}$ and $d=10 \mathrm{~cm}$ are about $4-8$ nm. ${ }^{16}$ The sizes of the $\mathrm{AlQ}_{3}$ nanoparticles prepared in this study under the same condition are about 50-100 $\mathrm{nm}$. These results agree with the prediction.

$\mathrm{AlQ}_{3}$ powder may exist in a variety of phases, such as $\alpha$, $\beta$, and $\gamma$ phases, ${ }^{17,18}$ and their $\mathrm{x}$-ray diffraction (XRD) patterns are quite different after evaporation. From the XRD patterns illustrated in Fig. 4, the $\mathrm{AlQ}_{3}$ powder has good crystallinity before evaporation, but the nanoparticles become amorphous after sublimation. To check if the $\mathrm{AlQ}_{3}$ molecules are decomposed during evaporation, the chemical bondings of $\mathrm{AlQ}_{3}$ in the nanoparticles, thin film, and original powder were examined by Fourier-transform infrared spectrum (FTIR). The FTIR test samples were prepared by growing $\mathrm{AlQ}_{3}$ nanoparticles directly on a potassium bromide (KBr) pellet. A thin film of thickness $1200 \AA$ was also deposited on another $\mathrm{KBr}$ pellet at a pressure of 1.33 $\times 10^{-3} \mathrm{~Pa}$ and a deposition rate of 3 to $5 \AA$ /s controlled by a quartz monitor. Except for the peaks due to $\mathrm{CO}_{2}$ and $\mathrm{H}_{2} \mathrm{O}$, all the other absorption peaks between 600 and $2000 \mathrm{~cm}^{-1}$ 


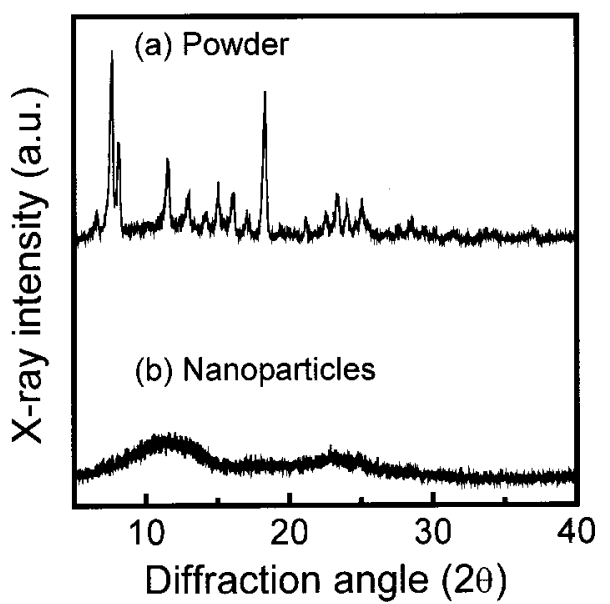

FIG. 4. XRD patterns of $\mathrm{AlQ}_{3}$ : (a) powder before evaporation and (b) nanoparticles of the size $50-100 \mathrm{~nm}$.

are found in good agreement with the reported results. ${ }^{19}$ The absorption peaks of the nanoparticles and the thin film are consistent with those of the $\mathrm{AlQ}_{3}$ powder, indicating that the bonding of the molecule is preserved even after evaporation at $410^{\circ} \mathrm{C}$.

The PL spectra were obtained by excitating with a $\mathrm{He}-\mathrm{Cd}$ laser of a wavelength $325 \mathrm{~nm}$ and a power $50 \mathrm{~mW}$. The $\mathrm{He}-\mathrm{Cd}$ laser beam was focused on an area of about $1 \mathrm{~mm}^{2}$ to ensure that all the samples had a similar irradiated area. The PL spectra of the $\mathrm{AlQ}_{3}$ nanoparticles with diameters of 50-100 and 100-300 nm are shown in Figs. 5(a) and 5(b), respectively. In addition, that of an $\mathrm{AlQ}_{3}$ thin film of a thickness $1200 \AA$ is also displayed in Fig. 5(c) for reference. The PL peaks show the same maximum intensity at about $5380 \AA$ and are broadened ranging from 4500 to $7000 \AA$. The

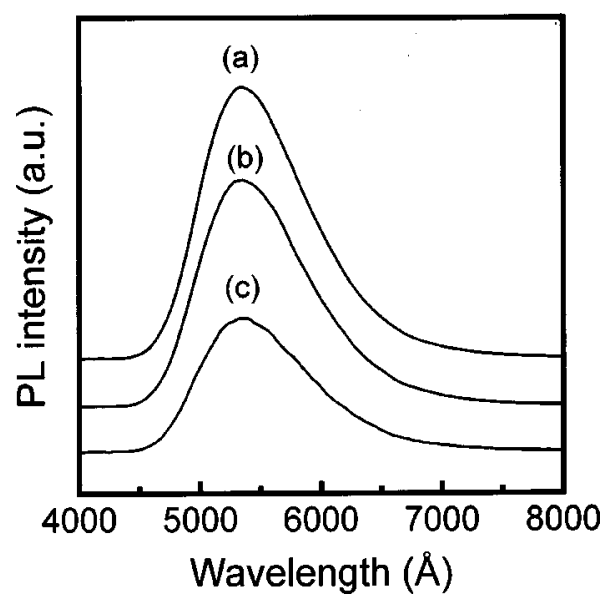

FIG. 5. PL spectra of $\mathrm{AlQ}_{3}$ : (a) nanoparticles of the size 50-100 nm, (b) nanoparticles of the size $100-300 \mathrm{~nm}$, and (c) thin film of a thickness 1200 $\AA$. maximum PL intensities of the nanoparticle samples are higher than that of the thin film, and the maximum PL intensity increases as the particle size decreases. It is generally agreed that the specific surface area increases as the particle size decreases. ${ }^{14,15}$ For ultrafine particles, they have very large specific surface area and surface energy. The larger specific surface area of the smaller nanoparticles increases the optical absorption and further enhances the intensity of luminescence. It is noted that the chemical bonding and surface morphology of the $\mathrm{AlQ}_{3}$ nanoparticles are preserved after aging in air at room temperature for more than one week, according to the FTIR spectra and FEGSEM images. However, degradation of the PL intensity occurs, and the degradation is more pronounced for the nanoparticles than for the thin film due to the larger specific surface area.

In summary, $\mathrm{AlQ}_{3}$ nanoparticles of the average size varying from 50 to $500 \mathrm{~nm}$ were synthesized by vapor condensation. The nanoparticles are spherical and have an amorphous structure. The chemical bonding is preserved after evaporation. The larger specific surface area of smaller nanoparticles increases the optical absorption and further enhances the intensity of photoluminescence. Related work will be of great interest for future study.

This work was supported by the National Science Council of ROC under Contract No. NSC-91-2215-E-002-024 and Ministry of Education of ROC under Contract No. A-91-EFA04-1-4.

${ }^{1}$ H. W. Kroto, J. R. Heath, S. C. O'Brien, R. F. Curl, and R. E. Smalley, Nature (London) 318, 162 (1985).

${ }^{2}$ S. Iijima, Nature (London) 354, 56 (1991).

${ }^{3}$ S. Fan, M. G. Chapline, N. R. Franklin, T. W. Tombler, A. M. Cassell, and H. Dai, Science 283, 512 (1999).

${ }^{4}$ R. F. Service, Science 271, 920 (1996).

${ }^{5}$ W. C. W. Chan and S. Nie, Science 281, 2016 (1998).

${ }^{6}$ O. B. Shchekin and D. G. Deppe, Appl. Phys. Lett. 80, 3277 (2002).

${ }^{7}$ X. Duan, Y. Huang, Y. Cui, J. Wang, and C. M. Lieber, Nature (London) 409, 66 (2001).

${ }^{8}$ M. Miyamura, K. Tachibana, and Y. Arakawa, Appl. Phys. Lett. 80, 3937 (2002).

${ }^{9}$ J. C. Kim, H. Rho, L. M. Smith, H. E. Jackson, S. Lee, M. Dobrowolska, J. L. Merz, and J. K. Furdyna, Appl. Phys. Lett. 73, 3399 (1998).

${ }^{10}$ R. A. Budiman and H. E. Ruda, J. Appl. Phys. 88, 4586 (2000).

${ }^{11}$ C. W. Tang and S. A. VanSlyke, Appl. Phys. Lett. 51, 913 (1987).

${ }^{12}$ A. Noy, A. E. Miller, J. E. Klare, B. L. Weeks, B. W. Woods, and J. J. DeYoreo, Arch. Nano Lett. 2, 109 (2002).

${ }^{13}$ J. H. Lim and C. A. Mirkin, Adv. Mater. 14, 1474 (2002).

${ }^{14}$ C. G. Granqvist and R. A. Buhrman, J. Appl. Phys. 47, 2200 (1976).

${ }^{15}$ N. Wada, Jpn. J. Appl. Phys. 7, 1287 (1968).

${ }^{16}$ H. S. Chen, J.-J. Chiu, and T.-P. Perng, Mater. Phys. Mech. 4, 62 (2001).

${ }^{17}$ M. Brinkmann, G. Gadret, M. Muccini, C. Taliani, N. Masciocchi, and A. Sironi, J. Am. Chem. Soc. 122, 5147 (2000).

${ }^{18}$ L. S. Sapochak, A. Padmaperuma, N. Washton, F. Endrino, G. T. Schmett, J. Marshall, D. Fogarty, P. E. Burrows, and S. R. Forrest, J. Am. Chem. Soc. 123, 6300 (2001).

${ }^{19}$ M. D. Halls and H. B. Schlegel, Chem. Mater. 13, 2632 (2001). 
Applied Physics Letters is copyrighted by the American Institute of Physics (AIP). Redistribution of journal material is subject to the AIP online journal license and/or AIP copyright. For more information, see http:/ojps.aip.org/aplo/aplcr.jsp

Copyright of Applied Physics Letters is the property of American Institute of Physics and its content may not be copied or emailed to multiple sites or posted to a listserv without the copyright holder's express written permission. However, users may print, download, or email articles for individual use. 